\title{
Concomitant BRAF Mutation in Hairy Cell Leukemia and Papillary Thyroid Cancer: Case Report
}

\author{
Shehab F. Mohamed ${ }^{a}$ Feryal Helmi $^{b}$ Susanna El-Akiki ${ }^{b}$ Halima El Omria \\ Abdulqadir Nashwan ${ }^{a}$ Mohamed A. Yassin ${ }^{a}$ \\ aHematology Department, National Center for Cancer Research (NCCCR), Hamad Medical \\ Corporation (HMC), Doha, Qatar; 'b Department of Laboratory Medicine and Pathology, \\ Hamad Medical Corporation (HMC), Doha, Qatar
}

\section{Keywords}

BRAF $\cdot$ Hairy cell leukemia $\cdot$ Papillary thyroid cancer

\begin{abstract}
Hairy cell leukemia $(\mathrm{HCL})$ is rare type of leukemia. This neoplasm is well-known to present with pancytopenia and splenomegaly. $\mathrm{HCL}$ is associated with BRAF mutation in $100 \%$ of cases. It is also associated with hematological and oncological malignancies such as melanoma and papillary thyroid cancer. Although the association of both cancers $(\mathrm{HCL}$ and papillary thyroid cancer) with BRAF mutation is well established in the literature, as far as we know it has not been reported before in the same patient. Here we report 48-year-old male diagnosed with $\mathrm{HCL}$ and papillary thyroid cancer and who is BRAF positive in both diagnostic tissues.
\end{abstract}

\section{Background}

Hairy cell leukemia (HCL) is an uncommon but distinct form of mature B-cell neoplasm that originates from activated late B-cells. It represents only $2 \%$ of all adult lymphoid leukemia. Patients are predominantly middle-aged to elderly males with a median age of 50 years, and it is characterized by pancytopenia, monocytopenia, and is usually associated with massive splenomegaly.

HCL is associated with BRAF mutation in $100 \%$ of cases. It is also associated with hematological and oncological malignancies such as melanoma and papillary thyroid cancer with positive BRAF in $40 \%$ of cases. 


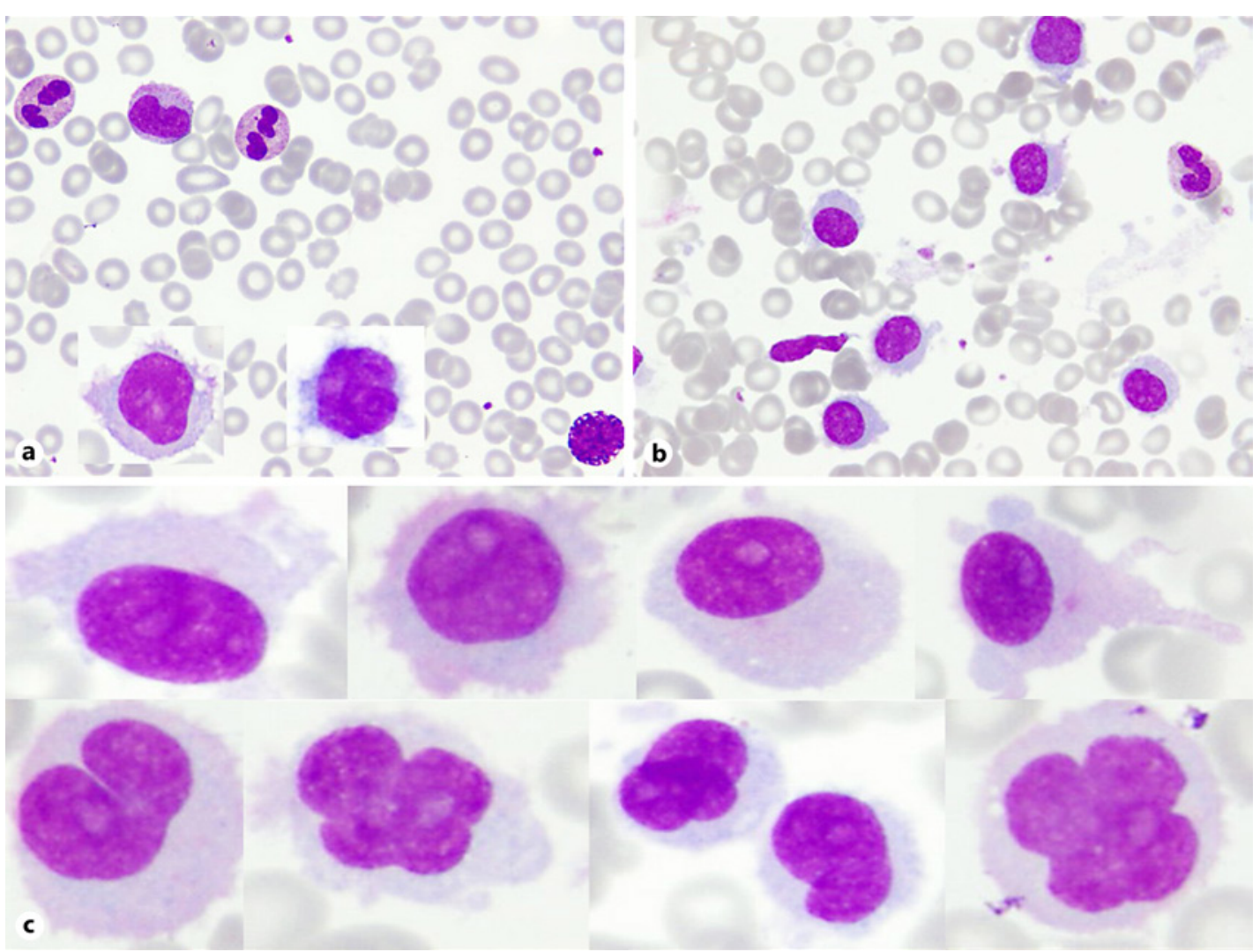

Fig. 1. a PB smear with two inserts of circulating hairy cells with oval to convoluted nucleus, homogeneous to dispersed chromatin, moderately abundant cytoplasm with shaggy hairy outline. b BM aspirate shows many hairy cells. Wright stain. $\times 1,000$. c Examples of hairy cells seen in the BM aspirate; medium to large with pleomorphic nuclear shape; round, oval, cleaved, convoluted or with irregular nuclear contour, mostly with homogenous to dispersed chromatin, many with prominent nucleolus. The cytoplasm is moderately abundant, faintly blue with shaggy or hairy outline.

BRAF mutation is a well-known finding in both HCL and papillary thyroid cancer but has never been reported in the same patient at the same time. Although the association of both cancers (HCL and papillary thyroid cancer) with BRAF mutation is well established in the literature, as far as we know this specific combination has not been previously reported in a single patient.

\section{Clinical Case}

A 48-year-old Lebanese male presented with bilateral hip pain and was found to have lytic bone lesions on both the x-ray and MRI. His complete blood count was normal and his abdominal ultrasound did not show any splenomegaly. Workup for myeloma was negative.

His peripheral blood (PB) showed mild normochromic normocytic anemia (Hb, $11.0 \mathrm{~g} /$ $\mathrm{dL}$ and mild thrombocytopenia $\left[129 \times 10^{3} / \mu \mathrm{L}\right]$ with a low normal leukocyte count $\left[4.2 \times 10^{3} /\right.$ $\mu \mathrm{L}]$ and absolute neutrophils $\left[2.3 \times 10^{3} / \mu \mathrm{L}\right]$ with monocytopenia $\left.\left[0.2 \times 10^{3} / \mu \mathrm{L}\right]\right)$.

Few circulating hairy cells were spotted on the screening of the peripheral smear with round to convoluted nuclei, homogeneous to dispersed chromatin, moderately abundant light blue cytoplasm with shaggy hairy outline, some with small nucleoli. 


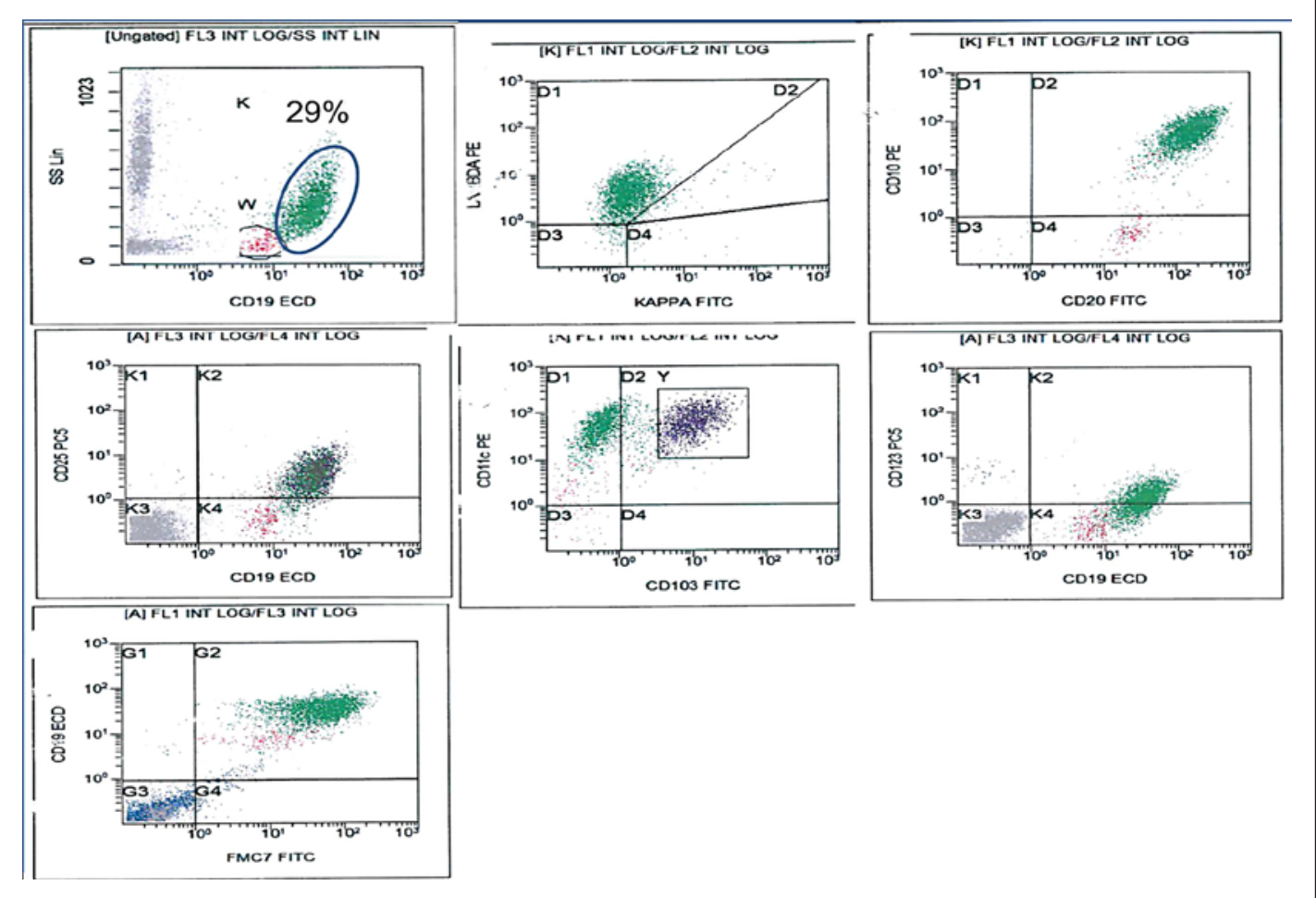

Fig. 2. Flow cytometry on BM aspirate revealed 29\% monotypic B-cells with heterogeneous high light side scatter with lambda light chain restriction and expressing bright CD19 and CD20 with coexpression of CD25, CD11c, CD103 on a subpopulation, CD123 and FMC7 and positive for CD10. The cells were also positive for CD79b and negative for CD5, CD23 (not shown).

Bone marrow (BM) aspirate smear showed extensive infiltration with hairy cells comprising approximately $60 \%$ with suppressed normal trilineal hemopoiesis. The cells were medium to large in size with rather pleomorphic nuclear shape; round or with irregular nuclear contour including cleaved or convoluted nucleus, mostly with homogenous to dispersed chromatin, many with prominent nucleolus. The cytoplasm was moderately abundant faintly blue with shaggy or hairy outline (Fig. 1).

Flow cytometry on BM aspirate revealed 29\% monotypic B-cells showing heterogeneous high light side scatter and expressing bright CD19 and CD20, CD79b with coexpression of FMC7, CD11c, CD25, and CD123 and included a subpopulation expressing CD103 with lambda light chain restriction. The cells were also positive for CD10 and negative for CD5 and CD23 (Fig. 2).

BM biopsy reflected marked hypercellularity ( 100\%), diffusely infiltrated by widely spaced lymphoid cells, with round, oval or irregular nuclei, many with a moderate amount of cytoplasm with a shaggy irregular outline. There was fried egg appearance in some areas with mildly increased fibrosis (grade $1 / 3$ score). By immunostaining the cells were positive for CD20, PAX5, DBA44, annexin A1, CD25, CD10, and many were positive for cyclinD1 (Fig. 3).

FISH analysis on marrow aspirate revealed a normal hybridization pattern for $t(11 ; 14)$ (q13;q32.3), CEP 12 (p11.1-q11.1), 13q34, ATM, 11q22.3, and TP53/CEP17, (17)(p13) with normal karyotype, 46,XY.

The patient was treated with 7 days of cladribine infusion. The patient's pain responded but he started to have a fever. A workup for pyrexia of unknown origin was done including 

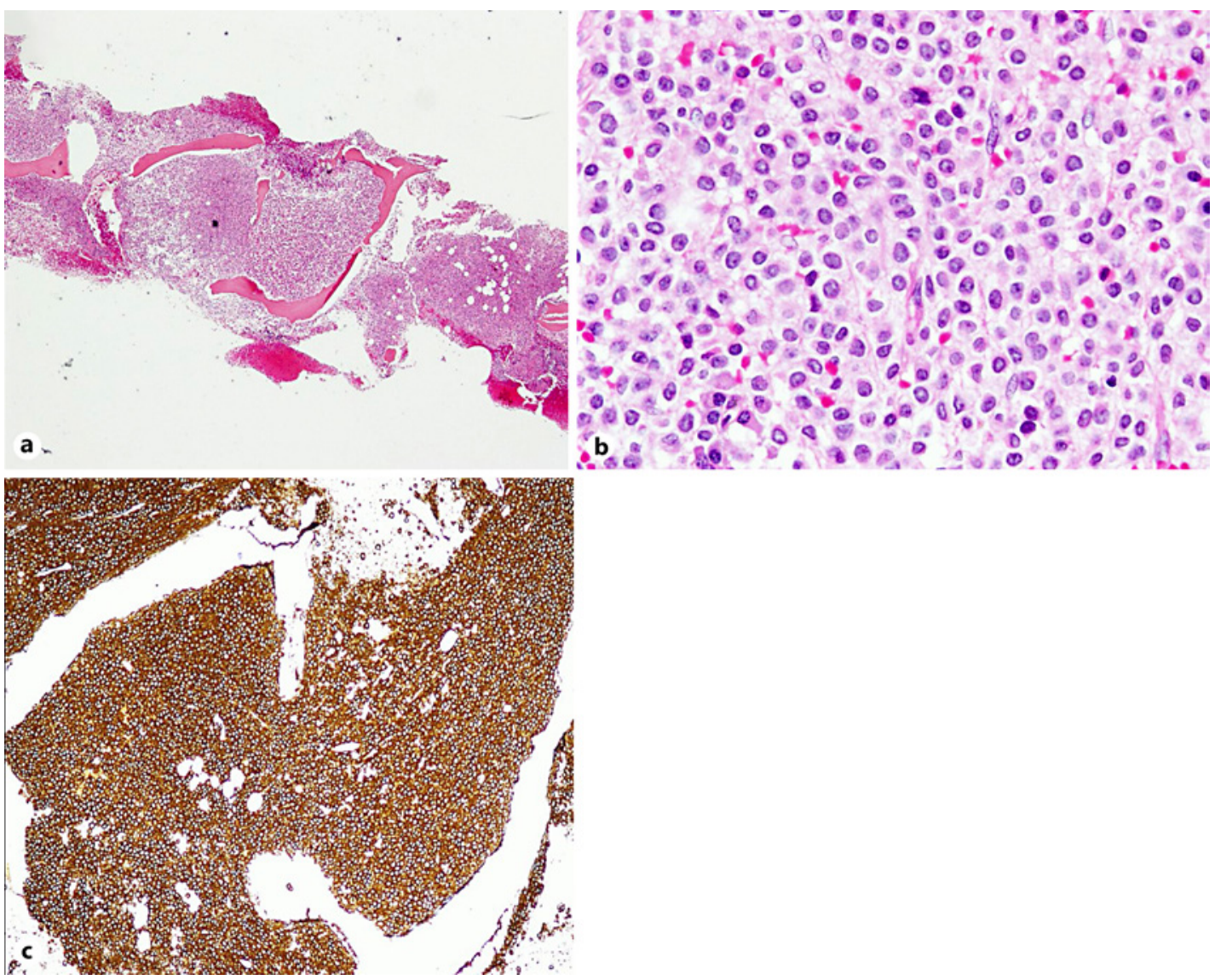

Fig. 3. a BM biopsy is markedly hypercellular ( 100\%). H\&E. $\times 100$. b Diffusely infiltrated by widely spaced lymphoid cells, with round, oval or irregular nucleus, many with a moderate amount of cytoplasm with a shaggy irregular outline. Fried egg appearance. c Cells are positive for CD20, DBA44. $\times 100$. Annexin A1 and CD25. $\times 200$.

positron tomography which showed abnormal uptake in the thyroid gland. The diagnosis of papillary thyroid cancer was subsequently established with thyroid (ultrasound) and fine needle aspiration. He was treated with total thyroidectomy followed by $30 \mathrm{mCi}$ of radioactive iodine (RAI). After diagnosis of papillary thyroid cancer, along with previous diagnosis of hairy cell leukemia, we decided to send BRAF mutation from both bone marrow tissue and thyroid tissue. Both samples came positive.

\section{Discussion}

HCL is an uncommon B-cell neoplasm; the clinical presentation in our case was unusual in several aspects: Firstly, the patient did not present with pancytopenia, which is common at presentation and is reported in $50-70 \%$ of patients with HCL [1]. Secondly, he did not have splenomegaly. Splenomegaly is the most common physical finding in HCL and is reported in $70-100 \%$ of cases [2]. Thirdly, HCL presenting as a lytic lesion is very uncommon. There is a previous case report of 4 patients with HCL without splenomegaly who also had lytic bone lesions [3]. 
Skeletal complications can occur as a consequence of HCL, with a reported incidence of $3 \%$, and usually present about 20 months after the initial diagnosis, but initial presentation with skeletal abnormalities is very rare [4].

The most interesting finding in this case was: BRAF-positive in both papillary thyroid cancer and BM biopsy of HCL. According to our knowledge and the search of the literature, not a single case report of this association has been reported before.

A central advance in understanding the pathogenesis of this disease was the finding of the activating point mutation in the kinase-encoding $B R A F$ gene in all patients with classical HCL in 2011 using the whole-exome sequencing technique. The mutation results in the substitution of adenine for thymine at position 1799 in exon 15 of the BRAF that replaces valine (V) by glutamate (E) at amino acid 600 (BRAF V600E) [5].

Although the BRAF V600E mutation is frequently present in different neoplasms, such as melanoma, papillary thyroid cancer, non-small-cell lung cancer, colorectal cancer, and Langerhans cell histiocytosis (X), the presence of the BRAF V600E mutation within the lymphoid neoplasms was found to be highly specific for $\operatorname{HCL}[5,6]$ and testing for this mutation is particularly useful in differentiating classic HCL from other B-cell neoplasm with overlapping features, such as HCL variant.

The BRAF V600E mutation can be identified in fresh PB or BM samples, as well as on archival Romanowsky-stained PB and BM smears and paraffin-embedded BM trephine biopsy using Sanger sequencing or polymerase chain reaction-based techniques [5-8].

Mutation in BRAF (particularly V600E) in HCL remarkably increases the BRAF kinase activity, rendering the protein constitutively active, phosphorylating then of ERK as a monomer becomes either independent of upstream regulatory signals or in a RAS-independent manner leading to constitutive activation of the RAF-MEK-ERK signaling pathway and enhancing survival of leukemic hairy cells $[9,10]$, similar to what occurs in other BRAFmutated tumors such as papillary thyroid carcinomas.

It is noteworthy that other BRAF mutations outside exon 15 were rarely reported as exon $11 \mathrm{~F} 468 \mathrm{C}$ and D449E mutations [11].

\section{Conclusion}

We emphasize the importance of having a high index of suspicion when considering a diagnosis of HCL based on histomorphological features even in the absence of classical clinical features. It might be worthwhile detecting the BRAF mutation in BM in patients with HCL as this might be linked to other forms of cancers that are asymptomatic such as in our patient and leads to early detection and diagnosis.

\section{Acknowledgment}

We acknowledge the support of the Qatar National Library.

\section{Statement of Ethics}

Ethical approval has been obtained from Hamada Medical Research Center, and informed consent has been obtained according to our institute's committee (ABHATH).

Our research complies with guidelines for human studies and was conducted ethically in accordance with the World Medical Association Declaration of Helsinki. 


\section{Disclosure Statement}

The authors have no conflicts to declare.

\section{Funding Sources}

The publication of this article was funded by Qatar National Library.

\section{Author Contributions}

All authors contributed equally to this work.

\section{References}

1 Swerdlow SH, Campo E, Pileri SA, Harris NL, Stein H, Siebert R. The 2016 revision of the World Health Organization (WHO) classification of lymphoid neoplasms. Blood. 2016 May;127(20):2375-90.

2 Spedini P, Tajana M, Bergonzi C. Unusual presentation of hairy cell leukemia. Haematologica. 2000;85(5):548.

3 Venkatesan S, Purohit A, Aggarwal M, Manivannan P, Tyagi S, Mahapatra M. Unusual presentation of hairy cell leukemia: a case series of four clinically unsuspected cases. Indian J Hematol Blood Transfus. 2014;30(Suppl 1):413-7.

4 Lembersky BC, Ratain MJ, Golomb HM. Skeletal complications in hairy cell leukemia: diagnosis and therapy. ] Clin Oncol. 1988;6(8):1280-4.

5 Tiacci E, Trifonov V, Schiavoni G, Holmes A, Kern W, Martelli MP. BRAF mutations in hairy-cell leukemia. New Engl J Med. 2011;364(24):2305-15.

6 Laurini JA, Aoun P, Iqbal J, Chan W, Greiner TC. Investigation of the BRAF V600E mutation by pyrosequencing in lymphoproliferative disorders. Am J Clin Pathol. 2012;138(6):877-83.

7 Arcaini L, Zibellini S, Boveri E, Riboni R, Rattotti S, Varettoni M. The BRAF V600E mutation in hairy cell leukemia and other mature B-cell neoplasms. Blood. 2012;119(1):188-91.

8 Thomas C, Amanuel B, Finlayson J, Grieu-Iacopetta F, Spagnolo DV, Erber WN. BRAF mutation detection in hairy cell leukaemia from archival haematolymphoid specimens. Pathology. 2015;47(4):349-54.

9 Wan PT, Garnett MJ, Roe SM, Lee S, Niculescu-Duvaz D, Good VM. Mechanism of activation of the RAF-ERK signaling pathway by oncogenic mutations of B-RAF. Cell. 2004;116(6):855-67.

10 Tiacci E, Schiavoni G, Martelli MP, Boveri E, Pacini R, Tabarrini A. Constant activation of the RAF-MEK-ERK pathway as a diagnostic and therapeutic target in hairy cell leukemia. Haematologica. 2013 Apr;98(4):635-9.

11 Tschernitz S, Flossbach L, Bonengel M, Roth S, Rosenwald A, Geissinger E. Alternative BRAF mutations in BRAF V 600 E-negative hairy cell leukaemias. Br J Haematol. 2014;165(4):529-33. 